TRANSACTIONS OF THE

AMERICAN MATHEMATICAL SOCIETY

Volume 226, 1977

\title{
THREE-DIMENSIONAL MANIFOLDS WITH FINITELY GENERATED FUNDAMENTAL GROUPS
}

BY

\author{
ROBERT MESSER
}

\begin{abstract}
Recent results of G. P. Scott and T. W. Tucker indicate that a three-dimensional manifold with a finitely generated fundamental group is, in various senses, close to being compact. In this paper the structure of such a manifold $M$ is described in terms of a certain compact, incompressible submanifold of $M$. This result is used to show that the product of $M$ with the real line is essentially the interior of a compact 4-manifold. Finally, when $M$ is $P^{2}$-irreducible, a necessary and sufficient condition is given for $M$ to be homeomorphic to the complement of a closed subset of the boundary of a compact 3-manifold.
\end{abstract}

Introduction. Suppose $M$ is an open, connected 3-manifold, $\pi_{1}(M)$ is finitely generated, $M$ contains no infinite collection of disjoint fake 3-cells, and $M$ contains no 2-sided projective planes. The first theorem of this paper describes $M$ in terms of a certain compact, incompressible submanifold $S$. Specifically, any compact subset of $M$ can be engulfed by first adding to $S$ a punctured collar on $\partial S$ and then attaching certain 1-handles.

The main result of $\$ 2$ uses this structure theorem to show that $M \times \mathbf{R}$ is homeomorphic to $(U \cup(C-P)) \times \mathbf{R}$ where $U$ is homeomorphic to a compact submanifold of $M, C$ is an open collar on $\partial U$, and $P$ is a tame, closed, 0 dimensional subset of Int $(C)$. In particular, if $\pi_{2}(M) \cong(0)$, then $P$ $=\varnothing$ and $M \times \mathbf{R} \cong \operatorname{Int}(U) \times \mathbf{R} \cong$ Int $(U \times I)$. It also follows that $M$ is homotopy equivalent to the interior of a compact 3-manifold with a tame, closed, 0-dimensional subset deleted.

83 contains an apparent improvement of Tucker's result [19]. A connected, $P^{2}$-irreducible 3-manifold is homeomorphic to the complement of a closed subset of the boundary of a compact 3-manifold if and only if for any polyhedral simple closed curve, the fundamental group of the complement of the curve is finitely generated.

This paper presents the results of [10] and contains the details of the research announcement [11]. D. R. McMillan suggested the main questions

Received by the editors July $14,1975$.

AMS (MOS) subject classifications (1970). Primary 55A05, 57A10, 57A65; Secondary 57A15, 57C99. 
this paper attempts to answer. His help and encouragement were essential to its completion.

The book by Rourke and Sanderson [12] will be the general reference for facts about piecewise linear topology. The book by Massey [5] will be the general reference for facts about 2-manifolds, covering spaces, fundamental groups, Van Kampen's theorem, and group theory. Results about incompressible surfaces in a product of a closed surface with a closed interval can be found in the article by Haken [4].

Most of the spaces and maps that we will consider are intended to be in the piecewise linear category. An n-manifold is a separable metric space such that each point has a neighborhood homeomorphic to an $n$-cell. A submanifold of an $n$-manifold will also be assumed to be $n$-dimensional. A surface is a connected 2-manifold. A 2-manifold $F$, properly embedded in a 3-manifold, is compressible if and only if either $F$ is a 2-sphere that bounds a 3-cell, or there is a disk $D$ such that $D \cap F=\partial D$ but $\partial D$ does not bound a disk in $F$. Otherwise $F$ is incompressible. A submanifold of a 3-manifold is incompressible if and only if it is either a 3-cell or has incompressible boundary. A fake 3-cell is a compact 3-manifold that is homotopy equivalent to a 3-cell, but is not homeomorphic to a 3-cell. The unit interval $[0,1]$ will be denoted by $I$, the interval $[-i, i]$ by $I_{i}$. A punctured product in an open 3-manifold $M$ between a component of the boundary of a submanifold $S$ and a closed surface $F$ disjoint from $S$ is a submanifold $P$ in $M-\operatorname{Int}(S)$, homeomorphic to $F \times I-\operatorname{Int}\left(B^{3}\right)$ where $B^{3}$ is a union of disjoint 3-cells in Int $(F \times I)$, such that under the homeomorphism, $F \times\{0\}$ corresponds to $F$ and $F \times\{1\}$ corresponds to $P \cap \partial S$. A punctured collar on $\partial S$ is a submanifold in $M-$ Int $(S)$ such that each component is a punctured product.

1. The structure of open 3-manifolds.

Definition. Suppose $M$ is an open, connected 3-manifold. A nucleus of $M$ is a compact submanifold $S$ such that for any compact subset $X$ of $M$ and any compact, incompressible submanifold $Y$ with $S \subseteq Y$, there is a compact submanifold $T$ with the following properties:

$Y \subseteq \operatorname{Int}(T)$,

$T-\operatorname{Int}(S)$ is a punctured collar on $\partial S$, and

$X$ is contained in the interior of the submanifold obtained by attaching certain 1-handles to $\partial T$.

The following theorem is modeled after a similar result of McMillan [8].

THEOREM 1. Suppose $M$ is an open, connected 3-manifold, $\pi_{1}(M)$ is finitely generated, $M$ contains no infinite collection of disjoint fake 3-cells, and $M$ contains no 2-sided projective planes. Then $M$ has an incompressible nucleus.

In Lemmas 1, 2 and 3, $M$ denotes a 3-manifold satisfying the hypotheses of 
Theorem 1. This theorem follows directly from Lemmas 1 and 3.

LEMMA 1. There is a compact submanifold $S$ of $M$ satisfying the following properties:

(1) Punctured 3-cells are the only compact, connected submanifolds of $M$ - Int(S) that are bounded by 2-spheres.

(2) $S$ is incompressible in $M$.

(3) Any indecomposable non-infinite-cyclic subgroup of $\pi_{1}(M)$ is conjugate to a subgroup of the fundamental group of a component of $S$.

(4) No component of $M-\operatorname{Int}(S)$ is compact.

(5) $S$ is not contained in any compact, incompressible submanifold of $M$ with fewer non-2-sphere boundary components.

(6) Every 2-sphere in $M-S$ separates the component of $M-\operatorname{Int}(S)$ containing it, and one of the resulting complementary domains contains no component of $\partial S$.

Proof OF LEMMA 1. By one of the properties of $M$ there is a finite collection of disjoint fake 3-cells in $M$ such that in the complement of these there are no additional fake 3-cells. In the complement of these fake 3-cells we can find a maximal collection of disjoint, compact, non-simply-connected submanifolds that are bounded by 2-spheres. In fact the number of such objects cannot exceed the the number of factors in an irreducible free-product decomposition of $\pi_{1}(M)$. Let $Q$ be the union of the objects in the above two collections.

Since $\pi_{1}(M)$ is finitely generated, Scott's theorem [13] shows that it is finitely presented. Find loops at a common base-point that represent the generators of a finite presentation of $\pi_{1}(M)$. Corresponding to the relators, find singular disks bounded by products of the appropriate loops. Let $S^{\prime}$ be a regular neighborhood of the union of these loops, singular disks, and $Q$. Then $S^{\prime}$ satisfies properties (1) and (3). However, since the map $\pi_{1}\left(S^{\prime}\right) \rightarrow \pi_{1}(M)$ induced by inclusion may not be a monomorphism, we should interpret property (3) as follows. Let $G$ be any indecomposable, non-infinite-cyclic subgroup of $\pi_{1}(M)$. There is a homomorphism of $G$ into $\pi_{1}\left(S^{\prime}\right)$ such that the composition of this map with the map $\pi_{1}\left(S^{\prime}\right) \rightarrow \pi_{1}(M)$ induced by inclusion is equal to conjugation of $G$ in $\pi_{1}(M)$. Notice that the map of $G$ into $\pi_{1}\left(S^{\prime}\right)$ must be a monomorphism.

To attain property (2) we will, whenever possible, modify $S^{\prime}$ by adding 2 handles and cutting 1-handles along compressing disks of the boundary of the resulting manifold. Properties (1) and (3) are clearly maintained when a 2handle is added. Since $\partial Q$ is a collection of 2-spheres, any disk used in cutting a 1-handle can be chosen to miss $\partial Q$. Thus property (1) can be maintained when a 1-handle is cut. Property (3) is also maintained when a 1-handle is cut. Indeed, before a 1-handle is cut, the fundamental group of the component involved is a free product $H * K$ where $H$ and $K$ are the funadmental groups 
of the resulting two components in the case the cut separates the component, and $K \cong \mathbf{Z}$ in the case the cut does not separate. In either case the Kurosh subgroup theorem implies that the map of $G$ into $H * K$ can be followed by a suitable conjugation of $H * K$ so that the image of $G$ is contained in either $H$ or $K$. Hence the above interpretation of property (3) holds for the manifold resulting from the cut.

If the resulting submanifold has punctured 3-cell components, we can add 1-handles to join these components to each other and to any other components. This will yield a submanifold satisyfing properties (1), (2) and (3). By adding any compact complementary domains, we see that property (4) can also be satisfied.

Choose $S$ so that it also satisfies the minimality condition (5) and so that among compact submanifolds of $M$ with properties (1), (2), (3), (4) and (5), the number of components of $S-Q$ is minimal. Then $S$ will also satisfy property (6). For if a 2-sphere in $M-S$ did not separate the component of $M-\operatorname{Int}(S)$ containing it, a regular neighborhood of the union of the 2-sphere and an appropriate transverse simple closed curve would be a compact, non-simplyconnected submanifold in $M-Q$ that is bounded by a 2-sphere. Similarly, if a 2-sphere in $M-S$ has components of $\partial S$ in both complementary domains, we could find a spanning arc of $M-\operatorname{Int}(S)$ that intersects the 2-sphere transversely and in a single point. The endpoints of the arc must be in the same component of $S-Q$, or else we could add to $S$ a regular neighborhood of the union of the arc and the 2-sphere. Also add the compact complementary domain if one is created. This will reduce the number of components of $S-Q$ while maintaining properties (1) through (5). Thus the arc can be completed in $S-Q$ to a simple closed curve. As above this would create a compact, nonsimply-connected submanifold in $M-Q$ that is bounded by a 2-sphere.

Lemma 2. Suppose $S$ is a compact submanifold of $M$ satisfying properties (2) and (3) of Lemma 1. Suppose $F$ is a closed, 2-sided, incompressible surface in $M$, $F$ is not a 2-sphere, and $F$ is disjoint from $S$. Then there is a punctured product $P$ between $F$ and a component of $\partial S$. Furthermore, if $E$ is a closed, incompressible 2-manifold in $M$ disjoint from $F \cup \partial S$, then $P$ can be chosen with the additional property that $E \cap \partial P=\varnothing$.

Proof of Lemma 2. Since $F$ is incompressible, we can consider $\pi_{1}(F)$ as a subgroup of $\pi_{1}(M)$. Since $\pi_{1}(F)$ is indecomposable and non-infinite-cyclic, it is conjugate to a subgroup of the fundamental group of some component of $S$. That is, there is a path $\gamma$ from the base-point of $F$ to the base-point of some component of $S$ such that for any loop $\alpha$ in $F$, the product $\gamma^{-1} \alpha \gamma$ is homotopic to a loop in $S$.

We want a map $f:(F \times I)-\operatorname{Int}\left(B^{3}\right) \rightarrow M$, where $B^{3}$ is a union of disjoint 3-cells in $\operatorname{Int}(F \times I)$, such that for all $x \in F, f(x, 1) \in \operatorname{Int}(S), f(x, 0)=x$, 
and in a neighborhood of $F \times\{0\}$, there are no other points of $f^{-1}(F)$.

Let $\alpha_{1}, \ldots, \alpha_{n}$ be simple closed curves in $F$ such that for $i \neq j, \alpha_{i} \cap \alpha_{j}$ is the base-point of $F$, and after cutting along $\alpha_{1}, \ldots, \alpha_{n}, F$ is reduced to a disk. Use the homotopies between the $\gamma^{-1} \alpha_{i} \gamma$ and corresponding loops in $S$ to define $f$ on $\left(\cup_{i=1}^{n} \alpha_{i}\right) \times I$ in $F \times I$ with the properties mentioned above. Extend $f$ to a regular neighborhood $N$ of

$$
\left(\left(\bigcup_{i=1}^{n} \alpha_{i}\right) \times I\right) \cup(F \times\{0\})
$$

maintaining these properties. Since $N \cap(F \times\{1\})$ is a regular neighborhood of $\left(\cup_{i=1}^{n} \alpha_{i}\right) \times\{1\}$, we need to define $f$ on the remaining disk of $F \times\{1\}$. But the boundary of this disk is also the boundary of the disk

$$
\operatorname{cl}(\partial N-\partial(F \times I)) \text {. }
$$

Thus we see that the image of this common boundary is a loop in $\operatorname{Int}(S)$, which is trivial in $M$. Since $\partial S$ is incompressible, this loop also bounds a singular disk in $\operatorname{Int}(S)$. Use this singular disk to extend $f$ to a regular neighborhood of $F \times\{1\}$. This leaves the interior of a single 3-cell in $\operatorname{Int}(F \times I)$ on which we have not defined $f$.

We will next modify $f$ to obtain the additional property that $f^{-1}(F \cup \partial S)$ is a collection of disjoint closed surfaces that are incompressible in $F \times I$. By general position we can assume that $f^{-1}(F \cup \partial S)$ is a collection of disjoint, properly embedded surfaces. Any boundary curve of $f^{-1}(F \cup \partial S)$ will bound a disk on the boundary of a 3-cell puncture. Thus the image of this curve will be trivial in $M$ and hence also in the component of $F \cup \partial S$ containing it. Therefore we can add a 2 -handle in the puncture along this curve and extend $f$. This process can be repeated, each time adding a disk to $f^{-1}(F \cup \partial S)$ to eliminate a boundary component of $f^{-1}(F \cup \partial S)$ while creating another 3cell puncture. Eventually we will attain the property that $\partial\left(f^{-1}(F \cup \partial S)\right)$ $=\varnothing$.

Suppose $f^{-1}(F \cup \partial S)$ is compressible in $F \times I$. Eliminate any 2-sphere component that occurs now or in the following construction by removing the interior of a punctured 3-cell containing it. Let $D_{0}$ be a compressing disk in $F \times I$. We can assume $D_{0}$ misses the punctures. In a neighborhood of $D_{0}$ we will perform a modification of the surgery used by Stallings in [17] and create three new 3-cell punctures to compress the surface. Let $D_{-}$and $D_{+}$be compressing disks on opposite sides of $D_{0}$ but parallel to it. One of the new 3cell punctures is a regular neighborhood of $D_{0}$ as indicated in Figure 1. The other two new 3-cell punctures are regular neighborhoods of points near the centers of $D_{-}$and $D_{+}$but on the sides opposite $D_{0}$. Consider the regular neighborhoods of $D_{-}$and $D_{+}$that meet the 3-cell punctues in disks in their boundaries as indicated in Figure 1. 


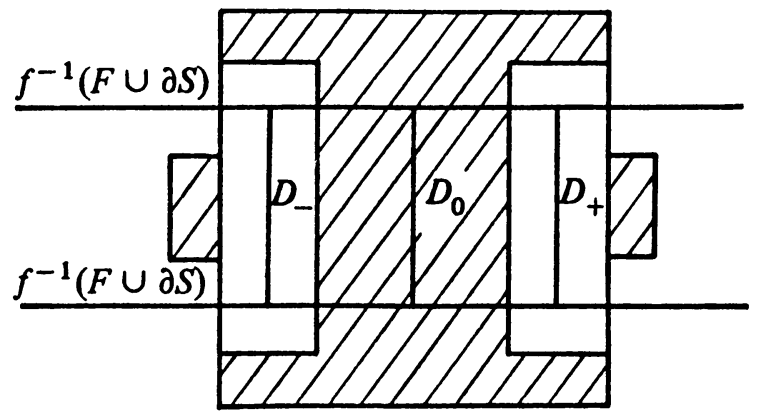

FigURE 1

We will redefine $f$ on these neighborhoods to replace the annulus of $f^{-1}(F \cup \partial S)$ between $\partial D_{-}$and $\partial D_{+}$with $D_{-} \cup D_{+}$. Each of these neighborhoods has an open annulus in its boundary that is not in the 3-cell punctures. These annuli are transverse to $f^{-1}(F \cup \partial S)$, and we can assume that $f$ maps these transverse to $F \cup \partial S$. Thus we can use the singular disks in $F \cup \partial S$ bounded by $f \mid \partial D_{-}$and $f \mid \partial D_{+}$to redefine $f$ on $D_{-} \cup D_{+}$, and then use the product structure of a neighborhood of $F \cup \partial S$ to extend the map to the rest of the neighborhoods.

After we have applied these techniques to make $f^{-1}(F \cup \partial S)$ incompressible in $F \times I$, we know that the components of $f^{-1}(F \cup \partial S)$ are parallel to $\partial(F \times I)$ (see Haken's lemma on p. 91 of [4]). We want to consider only the restriction of $f$ to the region between the highest component of $f^{-1}(F)$ and the next higher component of $f^{-1}(F \cup \partial S)$, which of course will be a component of $f^{-1}(\partial S)$. So now let

$$
f:(F \times I)-\operatorname{Int}\left(B^{3}\right) \rightarrow R
$$

denote this restriction, where $R$ is a complementary domain of $F \cup \partial S$. We have $f(F \times\{0\}) \subseteq F$ and $f(F \times\{1\}) \subseteq F^{\prime}$, a component of $\partial S$. Notice that

$$
(f \mid F \times\{0\})_{\sharp}: \pi_{1}(F \times\{0\}) \rightarrow \pi_{1}(F)
$$

and

$$
(f \mid F \times\{1\})_{\sharp}: \pi_{1}(F \times\{1\}) \rightarrow \pi_{1}\left(F^{\prime}\right)
$$

are monomorphisms. Notice also that since $F$ is 2-sided, one of the properties of $M$ eliminates the possibility that $F$ is a projective plane. Hence, by Tucker's version of Nielson's theorem [18], $f \mid F \times\{0\}$ and $f \mid F \times\{1\}$ are homotopic to covering maps. We can therefore assume that these restrictions are covering 
maps. Except in the cases that $F$ is a torus or a Klein bottle, these will be 1sheeted coverings.

Let $\alpha$ be a 2-sided, nonseparating simple closed curve in $F$. Some nonzero multiple of $\alpha$ lifts to a simple closed curve $\tilde{\alpha} \times\{0\}$ in $F \times\{0\}$. We can assume that the annulus $\tilde{\alpha} \times I$ is contained in $(F \times I)-B^{3}$. Thus $f(\tilde{\alpha} \times I)$ is a singular annulus between the multiple of $\alpha$ and a loop in $F^{\prime}$. Waldhausen's loop theorem [20] gives a nonsingular annulus $A$ from $\alpha$ to a simple closed curve in $F^{\prime}$. By modified surgery as before we can assume that $f^{-1}(A)$ is incompressible in $F \times I$, and hence it consists only of disjoint annuli, each from a component of $f^{-1}(\alpha)$ to a simple closed curve in $F \times\{1\}$. The restriction of $f$ to each of these annuli is homotopic to a covering map onto $A$. Hence we can assume that each such restriction is a covering map. Since $\alpha$ is 2-sided, we can apply Waldhausen's verticalizing Lemma 3.4 of [21]; thus we can also assume that the annuli are vertical in $F \times I$.

Let $F_{1} \times I$ be any component resulting from cutting $F \times I$ along these annuli. We obtain a map

$$
f_{1}:\left(F_{1} \times I\right)-\operatorname{Int}\left(B^{3}\right) \rightarrow R_{1}
$$

where $R_{1}$ is obtained from cutting $R$ along $A$.

Next we want to use a sequence of arcs that reduce $F_{1}$ to a disk to obtain disks properly embedded in $F_{1} \times I$ and in $R_{1}$, which, together with $A$, will define the 2-sphere boundary of a punctured product in $R$. Let $\beta$ be such an arc in $F_{1}$. Let $\tilde{\beta} \times\{0\}$ be a lift of $\beta$ to $F_{1} \times\{0\}$. We can assume that the disk $\tilde{\beta} \times I$ is contained in $\left(F_{1} \times I\right)-B^{3}$. Then $f_{1}(\tilde{\beta} \times I)$ gives a singular disk in $R_{1}$. The loop theorem [16] gives a nonsingular disk $D$ with $D \cap F_{1}=\beta$.

If $F$ is a torus or a Klein bottle, cutting $R$ along $A$ and then along $D$ will reduce $F$, and hence also $F^{\prime}$, to a disk. Thus a regular neighborhood in $R$ of $F \cup F^{\prime} \cup A \cup D$ will be a punctured product between $F$ and $F^{\prime}$.

On the other hand, if $F$ is of negative Euler characteristic, then in fact the restrictions of $f$ and $f_{1}$ to the respective 0-levels and 1-levels give homeomorphisms onto the respective surfaces in $\partial R$. Thus as we proceed with the modified surgery to make the inverse image of $D$ incompressible in $F_{1} \times I$, we will obtain a single disk with boundary equal to $\partial(\tilde{\beta} \times I)$. Verticalize this disk (see Lemma 3.4 of [21]). Cut along it, and in $R_{1}$ cut along $D$ to conclude another step in the reduction of $F$ and $F^{\prime}$ to disks. Continue this process with the other arcs of $F$. Eventually $F$, and hence also $F^{\prime}$, will be reduced to a disk. Thus a regular neighborhood in $R$ of the union of $F, F^{\prime}, A$, and these disks will be a punctured product between $F$ and $F^{\prime}$.

Suppose the boundary of the punctured product intersects $E$. Each component of the intersection must be a simple closed curve on the boundary of one of the punctures. Since $E$ is incompressible, each simple closed curve will 
bound a disk in $E$. Consider an innermost such disk in $E$. If this is outside the punctured product, add a 2-handle with the disk as core; if it is inside, remove a 2-handle with the disk as core. In either case we still have a punctured product but with one fewer curve of intersection between the boundary of the punctured product and $E$. Repeating this process will yield the desired punctured product.

LEMMA 3. Suppose $S$ is a compact submanifold of $M$ satisfying the six properties listed in Lemma 1 . Then $S$ is an incompressible nucleus of $M$.

Proof of Lemma 3. Let $X$ be a compact subset of $M$ and let $Y$ be a compact, incompressible submanifold with $S \subseteq Y$. Let $T^{\prime \prime}$ be a regular neighborhood of a simplicial neighborhood of $X \cup Y$. By cutting 1-handles and adding 2handles we want to form $T^{\prime}$ such that each component of $T^{\prime}$ is incompressible. Cut 1-handles so that the boundaries of the attaching disks are in $\partial T^{\prime \prime}$. Add 2handles so that the attaching annuli are in $\partial T^{\prime \prime}$ and the side disks intersect previously cut 1-handles in meridional disks. Since $Y$ is incompressible, we can assume that $Y \subseteq \operatorname{Int}\left(T^{\prime}\right)$. Let $T$ be a compact, incompressible submanifold of $M$ that has no compact complementary domains, contains $T^{\prime}$, and has a minimal number of non-2-sphere boundary components. Choose $T$ so that the 1-handles that were cut intersect $\partial T$ in meridional disks of the 1-handles. Finally, modify $T$ so that the number of components of $T$ has been minimized by adding a 1-handle in $M-\operatorname{Int}(T)$ missing the 1-handles that were cut, whenever it is possible to join a 2 -sphere component of $\partial T$ to some other component of $\partial T$.

Notice that any of the 1-handles that were cut to compress $\partial T^{\prime \prime}$ may be divided into several 1-handles by meridional disks of intersection with $\partial T$. Nevertheless by adding all of these 1-handles to $\partial T$ we will obtain a submanifold that contains $T^{\prime \prime}$. Hence $X$ will be contained in its interior (see $\mathrm{p}$. 65 of the paper [7] by McMillan for an alternative method of attaining this condition). We only need to show now that $T-\operatorname{Int}(S)$ consists of punctured products between corresponding components of $\partial S$ and $\partial T$.

Let $F_{1}, \ldots, F_{m}$ be the non-2-sphere components of $\partial T$ and let $E_{i}=\partial T$ $-F_{i}$. By Lemma 2 there is a punctured product $P_{1}$ between $F_{1}$ and a component of $\partial S$, such that $P_{1} \cap S$ is only this component of $\partial S$ and such that $E_{1} \cap \partial P_{1}=\varnothing$. Notice that we can modify the punctures so that no 2-sphere component of $E_{1}$ is contained in $P_{1}$. Furthermore, no non-2-sphere component of $E_{1}$ can be contained in $P_{1}$. Indeed, between any such component and either of $F_{1}$ or $P_{1} \cap S$, there would be a punctured product. Then as we go down through these surfaces starting from $P_{1} \cap S$, we would pass from $T$ to $M-T$ as we crossed the first component of $E_{1}$, and back into $T$ as we crossed the second or reached $F_{1}$. Between these two components of $\partial T$ there would be a punctured product which could be added to $T$. This would reduce the number 
of non-2-sphere boundary components and contradict our minimality assumption on the number of non-2-spheres in $\partial T$. It follows from this fact that $P_{1}$ is contained in $T$. Otherwise there would be an intersection with $E_{1}$ as the punctured product entered $T$ to reach $\partial S$. Since $T$ has no compact complementary domains, we can add to $P_{1}$ any 3 -cell complementary domains. It follows then that $\partial P_{1}$ is incompressible.

Now $S \cup P_{1}$ satisfies properties (2) and (3) of Lemma 1, so by Lemma 2 we obtain a punctured product $P_{2}$ between $F_{2}$ and a component of $\partial\left(S \cup P_{1}\right)$, such that $P_{2} \cap\left(S \cup P_{1}\right)$ is only this component of $\partial\left(S \cup P_{1}\right)$ and such that $E_{2} \cap \partial P_{2}=\varnothing$. Although Lemma 2 does not guarantee that $F_{1}$ is not a component of $\partial P_{2}$, the minimality condition on the number of non-2-spheres in $\partial T$ prevents this. As argued in the previous paragraph, we can assume that $P_{2}$ is contained in $T$, and that $\partial P_{2}$ is incompressible.

For each $F_{i}$ apply this process inductively in the complement of $S \cup P_{1}$ $\cup \cdots \cup P_{i-1}$. We finally obtain disjoint punctured products $P_{1}, \ldots, P_{m}$ in $T$ between the non-2-sphere components of $\partial T$ and corresponding components of $\partial S$.

Property (5) yields that there are no further non-2-sphere components of $\partial S$. Therefore

$$
\operatorname{cl}\left(T-\left(S \cup P_{1} \cup \cdots \cup P_{m}\right)\right)
$$

has only 2-sphere boundary components, namely,

$$
\left(\partial P_{1} \cup \cdots \cup \partial P_{m}\right)-(\partial S \cup \partial T)
$$

and the 2-spheres of $\partial S \cup \partial T$. By property (1) the components of

$$
\operatorname{cl}\left(T-\left(S \cup P_{1} \cup \cdots \cup P_{m}\right)\right)
$$

are punctured 3-cells. Consider one of these punctured 3-cells that has a boundary component in common with one of the punctured products $P_{i}$. By property (6), this 2-sphere boundary component will separate the component of $M-\operatorname{Int}(S)$ containing it and, since one of the resulting complementary domains contains $P_{i}$ and hence a component of $\partial S$, the other complementary domain can contain no components of $\partial S$. Therefore no other boundary component of this punctured 3-cell can intersect $S \cup P_{1} \cup \cdots \cup P_{m}$.

By joining these punctured 3-cells to $P_{1}, \ldots, P_{m}$, we have established that the non-2-sphere components of $\partial T$ and $\partial S$ are in one-to-one correspondence and that there are punctured product components of $T-\operatorname{Int}(S)$ between corresponding components of $\partial T$ and $\partial S$. We now need only see that the remaining components of $T-\operatorname{Int}(S)$ give the same kind of correspondence between 2-sphere components of $\partial S$ and $\partial T$. We already know that these components of $T-\operatorname{Int}(S)$ are punctured 3-cells. 
Recall in the construction of $T$ that 2-sphere components of $\partial T$ were joined to other components of $\partial T$ whenever possible. Thus for any 2-sphere component of $\partial T$, the punctured 3-cell of $T-\operatorname{Int}(S)$ containing it also contains a component of $\partial S$. Using property (6) as above shows that there is only one such component of $\partial S$. Hence this component of $T-\operatorname{Int}(S)$ is a punctured product between the chosen component of $\partial T$ and a 2-sphere component of $\partial S$. Repeat this argument for any remaining components of $\partial T$.

Since $S$ has no compact complementary domains, we have accounted for all the components of $\partial S$.

Theorem 2 is a converse of Lemma 3. It will be useful in the proofs of Theorems 3 and 4.

THEOREM 2. Suppose $S$ is a nucleus of an open, connected 3-manifold $M$ and either $S$ is a 3-cell or $\partial S$ is incompressible in $S$. Then $S$ satisfies the six properties listed in Lemma 1.

The condition that either $S$ is a 3-cell or $\partial S$ is incompressible in $S$ is necessary. For example the union of two disjoint 3-cells in $\mathbf{R}^{3}$ is a nucleus of $\mathbf{R}^{3}$. Also, a solid torus embedded as a core of an open solid torus is a nucleus of the open solid torus. In both of these examples property (2) of Lemma 1 is not satisfied.

Proof OF THEOREM 2. Let us consider a compact, connected submanifold of $M-\operatorname{Int}(S)$ that is bounded by 2 -spheres. This submanifold is contained in a punctured collar on $\partial S$, with 1-handles attached. It follows that the submanifold can only be a punctured 3-cell. Thus $S$ satisfies property (1).

Any component of $M-\operatorname{Int}(S)$ can be written as the union of a nested sequence of punctured collars with 1-handles attached. Such a union cannot be compact. Thus $S$ satisfies property (4).

By hypothesis and since we have verified property (4), the 2-spheres of $\partial S$ are incompressible unless $S$ is a 3-cell. Again by hypothesis, there is no compressing disk of $\partial S$ that is contained in $S$. Suppose there is a compressing disk of $\partial S$ in $M-\operatorname{Int}(S)$. This disk could be engulfed by first adding to $S$ a punctured collar on $\partial S$ and then attaching certain 1-handles. But each component of $\partial S$ is injective in the punctured collar, and therefore injective and hence incompressible in the punctured collar with the 1-handles attached. Thus such a compressing disk does not exist, and $S$ satisfies property (2).

As we saw in the proof of Lemma 1, it is easy to construct a compact submanifold of $M$ with property (3). We can engulf such a submanifold by first adding to $S$ a punctured collar on $\partial S$ and then attaching certain 1handles. We also know from the proof of Lemma 1 that property (3) is maintained as the 1-handles are removed. Since any loop in the union of $S$ and the punctured collar on $\partial S$ is homotopic to a loop in $S$, it follows that $S$ satisfies property (3). 
Suppose $S$ is contained in a compact, incompressible submanifold $S^{*}$ of $M$. There is a compact submanifold $T$ such that $S^{*} \subseteq \operatorname{Int}(T)$ and $T-\operatorname{Int}(S)$ consists of punctured products between corresponding components of $\partial S$ and $\partial T$. For any component of $\partial S$ that is not a 2-sphere, consider the component of $T-\operatorname{Int}(S)$ containing it. If all the components of $\partial S^{*}$ in this punctured product were 2-spheres, we could not have $S \subseteq S^{*} \subseteq T$. Thus we see that $\partial S^{*}$ has at least as many non-2-sphere components as $\partial S$ has. This gives property (5).

If property (6) fails, there would be a 2 -sphere in $M-\operatorname{Int}(S)$ and a spanning arc of $M-\operatorname{Int}(S)$ that intersects the 2 -sphere transversely and in a single point. Add to $S$ a regular neighborhood of the union of the arc and the 2-sphere. Also add the compact complementary domain if one is created. We obtain a compact, incompressible submanifold of $M$ that contains $S$. Thus we can add to $S$ a punctured collar on $\partial S$ to engulf this submanifold. The arc and the 2-sphere are in a single component of the punctured collar; so, in particular, the ends of the arc must be in the same component of $\partial S$. But then we could complete the arc in the punctured product to a simple closed curve that intersects the 2-sphere transversely and in a single point. This contradicts the fact that punctured products do not contain nonseparating 2 -spheres. Thus $S$ satisfies property (6).

Theorem 3 is a weak uniqueness theorem for incompressible nuclei. Since the intersection of a component of one incompressible nucleus with a component of another may consist of several components, each containing some essential bit of structure of the 3-manifold, this seems to be the best uniqueness result.

THEOREM 3. Suppose $M$ is an open, connected 3-manifold. Suppose $S$ and $S^{*}$ are incompressible nuclei of $M$. Then $S^{*}$ can be obtained from $S$ when the following sequence of steps is performed appropriately:

(1) Add to $S$ a punctured collar on $\partial S$.

(2) Attach 1-handles to the boundary of the resulting submanifold.

(3) For each of these 1-handles cut the submanifold along a disk having boundary equal to the boundary of a meridional disk of the 1-handle.

(4) Remove a punctured collar between the boundary of the resulting submanifold and $\partial S^{*}$.

COROLlary. Suppose $S$ is an incompressible nucleus of an open, connected 3manifold $M$. The number of non-2-sphere components of $\partial S$ of any given homeomorphism type is an invariant of $M$.

Proof of TheORem 3. There is a compact submanifold $T$ such that $T-\operatorname{Int}(S)$ is a punctured collar on $\partial S$ and by adding certain 1-handles to $\partial T$ we can create a submanifold $V$ with $S^{*} \subseteq$ Int $(V)$. Since $S$ satisfies the 
properties of Lemma 1, we can choose $T$ to have no compact complementary domains.

Choose a meridional disk for each of the 1-handles of $V$ and let $D$ denote the union of these disks. Notice that $\partial D \cap S^{*}=\varnothing$. We want to modify the interiors of these disks to obtain disks that are disjoint from $\partial S^{*}$, and hence also from $S^{*}$. We can assume that $D \cap \partial S^{*}$ is a collection of simple closed curves. Any such curve bounds a disk in $D$, so it also bounds a disk in $\partial S^{*}$. Thus we can consider a simple closed curve of $D \cap \partial S^{*}$ that bounds a disk in $\partial S^{*}$ containing no other intersection curves. Cut $D$ off near $\partial S^{*}$ along this disk. This reduces the number of intersection curves. Therefore by repeating this process, we will eventually obtain the desired disks.

Let $Y^{*}$ be the submanifold obtained by cutting $V$ along these disks. Then $S^{*} \subseteq \operatorname{Int}\left(Y^{*}\right)$. To see that $Y^{*}$ is incompressible, notice that each component of $Y^{*}$ has been obtained from a corresponding component of $T$ by adding and cutting 1-handles as indicated in the previous paragraph. Since $T$ has no compact complementary domains, neither does $Y^{*}$. Thus any 2-sphere of $\partial Y^{*}$ is incompressible unless it bounds a 3-cell component of $Y^{*}$. If $Y^{*}$ has a 3-cell component, the correspondence between components of $Y^{*}$ and $T$ shows that there is a component of $T$ bounded by a single 2-sphere. Hence there is a component of $S$ bounded by a single 2-sphere. By property (6) of Lemma 1, it follows that $S$ consists only of this component. Hence $Y^{*}$ is only this 3-cell. Consider now a simple closed curve in a non-2-sphere component of $\partial Y^{*}$. We can assume it is disjoint from the attaching disks of the 1-handles that were cut in forming $Y^{*}$. Thus the curve is also in $\partial T$. If it bounds a disk in $M$, it will also bound a disk in $\partial T$. Although the interior of this disk might contain attaching disks of 1-handles of $V$, we can replace each such attaching disk with the corresponding attaching disk in $\partial Y^{*}$ of a 1-handle that was cut in forming $Y^{*}$. This will give a disk in $\partial Y^{*}$ bounded by the simple closed curve.

Now then, there is a compact submanifold $T^{*}$ that consists of $S^{*}$ with a punctured collar on $\partial S^{*}$ and is such that $Y^{*} \subseteq \operatorname{Int}\left(T^{*}\right)$. Using again the fact that $Y^{*}$ is incompressible, we see that there is a punctured collar between $\partial Y^{*}$ and $\partial S^{*}$.

2. The product of an open 3-manifold with the real line. Whitehead's example [22] of a contractible, open 3-manifold shows that we cannot rely on homotopy properties to determine whether an open 3-manifold is the interior of a compact 3-manifold. Nevertheless, similar to McMillan's technique in [6] and [9] of showing that the product of the Whitehead example with $\mathbf{R}$ is the interior of a 4-cell, Theorem 4 shows that much of the difficulty is eliminated when we consider the product of an open 3-manifold with $\mathbf{R}$.

The following lemma will be used in Theorem 4 and Theorem 5 .

LeMMA 4. Suppose $M$ is a connected 3-manifold and $\pi_{1}(M)$ is finitely generated. 
Suppose $F$ is a 2-sided, incompressible 2-manifold properly embedded in $M$, the fundamental group of each component of $F$ is finitely generated, and only a finite number of components of $F$ are non-simply-connected. Then for any component $L$ of the 3-manifold obtained by cutting $M$ along $F, \pi_{1}(L)$ is finitely generated.

Proof of Lemma 4. Let $F_{1}, F_{2}, \ldots$ denote the components of cl $(\partial L-\partial M)$. These are the surfaces in $\partial L$ that result from cutting $M$ along $F$. For each $i \geqslant 1$ choose a base-point $x_{i} \in F_{i}$ and choose a path $e_{i}$ in $L$ from $x_{1}$ to $x_{i}$, with $e_{1}$ being the constant path at $x_{1}$. For each non-simply-connected component $F_{i}$, choose loops $f_{i 1}, \ldots, f_{i m_{i}}$ in $F_{i}$ based at $x_{i}$ that represent a finite set of generators of $\pi_{1}\left(F_{i}\right)$. For each simply-connected component $F_{i}$, let $f_{i 1}$ denote the constant loop at $x_{i}$. Let $g_{1}, \ldots, g_{m}$ be loops based at $x_{1}$ that represent a finite set of generators of $\pi_{1}(M)$. We can assume that the $g_{i}$ intersect $\partial L$ transversely and only at the points $x_{1}, x_{2}, \ldots$ Thus the points $x_{1}, x_{2}, \ldots$ divide the loops $g_{1}, \ldots, g_{m}$ into paths. Of these paths, let $h_{1}, \ldots, h_{n}$ denote those that are in $L$.

Now let $\gamma$ be any loop in $L$ based at $x_{1}$. Assume $\gamma \cap \partial L=\left\{x_{1}\right\}$. Consider the domain of the singular disk that defines a homotopy in $M$ between $\gamma$ and a product of the loops $g_{i}^{ \pm 1}$. Assume the singular disk is in general position with respect to $\partial L$. Since $F$ is 2 -sided and incompressible, we can eliminate simple closed curves resulting from intersections with $\partial L$. Thus we see that $\gamma$ is homotopic in $L$ to a product of paths $h_{j}^{ \pm 1}$ and loops in the components of $\partial L$ based at the chosen base-point. It follows that $\gamma$ is homotopic in $L$ to a product of loops of the forms $e_{i} h_{j}^{ \pm 1} e_{k}^{-1}$ and $e_{i} f_{i j}^{ \pm 1} e_{i}^{-1}$ for appropriate values of $i, j$ and $k$. Since there are only a finite number of such loops that are nontrivial, we see that $\pi_{1}(L)$ is finitely generated.

THEOREM 4. Suppose $M$ is an open, connected 3-manifold, $\pi_{1}(M)$ is finitely generated, and $M$ contains an incompressible nucleus. Assume $S$ is an incompressible nucleus of $M$ with a maximal number of non-simply-connected complementary domains. Then $M \times \mathbf{R}$ is homeomorphic to $(U \cup(C-P)) \times \mathbf{R}$ where $U$ is homeomorphic to a submanifold of $M$ obtained by adding certain 1-handles to $S$, $C$ is an open collar on $\partial U$, and $P$ is a tame, closed, 0 -dimensional subset of Int $(C)$. Specifically, any compact subset of $P$ can be written as the intersection of a nested sequence of unions of disjoint 3-cells in Int $(C)$.

Suppose $S$ and $S^{*}$ are incompressible nuclei of $M$ with $S \subseteq \operatorname{Int}\left(S^{*}\right)$. Then cl $\left(S^{*}-S\right)$ is a punctured collar on $\partial S$. Hence the fundamental group of a component of $M-$ Int $(S)$ is the free product of the fundamental groups of those components of $M-$ Int $\left(S^{*}\right)$ that it contains. By Lemma 4 the fundamental group of any component of $M-\operatorname{Int}(S)$ is finitely generated; so nontrivial free-product factorization cannot continue indefinitely. Hence it is without loss of generality that we can assume the incompressible nucleus $S$ has 
a maximal number of non-simply-connected complementary domains.

COROLLARY. In the situation stated in Theorem 4, suppose also that $\pi_{2}(M)$ $\cong(0)$. Then $P=\varnothing$ and $M \times \mathbf{R}$ is homeomorphic to Int $(U) \times \mathbf{R}$, which is homeomorphic to the interior of the compact 4-manifold $U \times I$.

The following corollary is an extension of Theorem 3.2 of [3].

COROLlary. Suppose $M$ is a connected 3-manijuld, $\pi_{1}(M)$ is finitely generated, $M$ contains no infinite collection of disjoint fake 3-cells, and $M$ contains no 2-sided projective planes. Then $M$ is homotopy equivalent to the interior of a compact 3manifold with a tame, closed, 0-dimensional subset deleted. The compact 3manifold is homeomorphic to a submanifold of $M$.

Proof. Since any manifold is homotopy equivalent to its interior, we need only establish the result when $M$ is an open 3-manifold. Theorem 1 implies the $M$ has an incompressible nucleus. Thus Theorem 4 applies to give the result.

COROLlary. Suppose $N$ is a closed, connected, orientable, irreducible, sufficiently large 3-manifold. Suppose $M$ is a noncompact covering space of $N$ associated with some finitely generated subgroup of $\pi_{1}(N)$. Then $M \times R$ is homeomorphic to the interior of the product of a compact 3-manifold with $I$.

Proof. By Theorem 8.1 of Waldhausen's paper [21], the universal cover of $N$ is irreducible. Hence $M$ is irreducible. The corollary follows from Theorem 1 and the first corollary to Theorem 4.

Proof of Theorem 4. Except near the very end of this proof, we will primarily be concerned with only a single component $L$ of $M-\operatorname{Int}(S)$. However, the constructions are to be carried out in each component of $M-\operatorname{Int}(S)$.

We want to construct sequences $T_{i}, U_{i}, V_{i}$ and $D_{i}$, where $i=1,2, \ldots$, with the following properties:

$T_{i}$ is a punctured collar in $L$ on $\partial L$.

No component of $\mathrm{cl}\left(L-T_{i}\right)$ is compact.

For $i \geqslant 1, T_{i+1}$ is also $T_{i}$ with a punctured collar in cl $\left(L-T_{i}\right)$ on $\partial T_{i}-\partial L$. $U_{i}$ is $T_{i}$ with 1 -handles attached so that $U_{i}$ is connected and the map $\pi_{1}\left(U_{i}\right) \rightarrow \pi_{1}(L)$ induced by inclusion is an isomorphism.

For $i \geqslant 1$, there is a one-to-one correspondence between the 1-handles of $U_{i}$ and the 1-handles of $U_{i+1}$ with corresponding 1-handles being homotopic. That is, for a core $\alpha$ of any 1-handle of $U_{i}$ we will be able to extend a core of the corresponding 1-handle of $U_{i+1}$ to the endpoints of $\alpha$ by arcs that are vertical with respect to the fiber structure of $T_{i+1}$, to form an arc $\beta$ that is homotopic in $L$ relative to $\partial \beta$, to $\alpha$. 
$V_{i}$ consists of $U_{i}$ with more 1-handles attached. These will be referred to as the extra 1-handles of $V_{i}$.

$\cup_{i=1}^{\infty} V_{i}=L$.

For $i \geqslant 1,\left(V_{i}-\partial L\right) \subseteq \operatorname{Int}\left(V_{i+1}\right)$ and $D_{i} \subseteq \operatorname{Int}\left(V_{i+1}\right)$.

$D_{i}$ is a collection of singular disks $d_{j}: D^{2} \rightarrow L$. Each $d_{j}$ determines a homotopy from the core of an extra 1-handle of $V_{i}$ to a spanning arc of $U_{i}$. The union of the images of these maps will also be denoted $D_{i}$.

$d_{j}^{-1}\left(V_{i}\right)$ consists of disks in Int $\left(D^{2}\right)$ that map to meridional disks of 1 handles of $V_{i}$, and of a collar on $\partial D^{2}$. This collar can be cut apart into a collection of disks that each meets $\partial D^{2}$ in an arc and such that one of the disks maps into the extra 1-handle while the others map into $U_{i}$.

The maps of $D_{i}$ are in general position with respect to each other, and the singular set of the union of these maps is such that:

no branch points map into $V_{i}$,

the image of each double line is an arc with one end on the core of an extra 1-handle of $V_{i}$, and

there are no triple points.

Let $B_{i}, i=1,2, \ldots$, be a sequence of compact subsets of $L$ such that $\cup_{i=1}^{\infty} B_{i}=L$.

Let us now proceed with the construction of $T_{i}, U_{i}, V_{i}$, and $D_{i}$. By Lemma 4 and Scott's result [13], $\pi_{1}(L)$ is finitely presented. Let $X_{1}$ equal the union of $B_{1}$, loops in $L$ that represent a finite set of generators of $\pi_{1}(L)$, and singular disks in $L$ corresponding to a finite set of relators for $\pi_{1}(L)$ with the given generators. We know that $X_{1}$ can be engulfed by first adding to $S$ a punctured collar on $\partial S$ and then attaching certain 1-handles to the boundary of the resulting submanifold. Let $T_{1}$ be the part of the punctured collar that is in $L$. Then $T_{1}$ is a punctured collar in $L$ on $\partial L$. Suppose that a component of cl $\left(L-T_{1}\right)$ is compact. Since $S$ satisfies property (5) of Lemma 1 , this component must have only 2 -sphere boundary components. By properties (1) and (6) this component must be a 3-cell, which can be added to $T_{1}$ to eliminate a puncture.

By adding certain 1-handles to $T_{1}$ we know we can obtain a submanifold $V_{1}^{\prime}$ of $L$ that contains $X_{1}$. We now apply Scott's construction [14] to $V_{1}^{\prime}$ to obtain a compact submanifold $U_{1}$ with $\pi_{1}\left(U_{1}\right) \rightarrow \pi_{1}(L)$ an isomorphism.

Stage 1 . We can make $\partial V_{1}^{\prime}$ incompressible by cutting off all the 1-handles that were added to $T_{1}$.

Stage 2. Map $L$ to the aspherical complex constructed on the basis of an irreducible free-product decomposition of $\pi_{1}(L)$. Consider the midpoints of the 1 -simplexes that join the pieces of this complex corresponding to the indecomposable factors of the decomposition of $\pi_{1}(L)$. We can assume the inverse image of these points is a 2-manifold, properly embedded in $L$. By surgery we can reduce the part of this 2-manifold in $T_{1}$ to incompressible 2-spheres. Each 
such 2-sphere bounds a punctured 3-cell in $T_{1}$. So by further surgery we can assume that each of the 2-spheres is parallel to a component of $\partial T_{1}-\partial L$. Then we can adjust the map to eliminate these remaining inverse image components in $T_{1}$. It follows that $T_{1}$ is unaffected by this stage.

Stage 3. This is not needed in Scott's proof or here.

Stage 4. Choose one component of $T_{1}$ for each non-infinite-cyclic factor of the decomposition of $\pi_{1}(L)$. Suppose that there is a component of $T_{1}$ that is not chosen. If such a component has only 2-sphere boundary components, the fact that $S$ satisfies property (6) of Lemma 1 implies that this component is all of $T_{1}$. It follows that $\pi_{1}(L)$ is a free group. We can complete the construction in this case by adding 1 -handles to $T_{1}$ in $L$, that represent a set of free generators of $\pi_{1}(L)$. This will yield $U_{1}$ with the desired properties. We can choose these 1handles to be disjoint from the 1-handles of $V_{1}^{\prime}$. Then the 1-handles of $V_{1}^{\prime}$ can be added to $U_{1}$ to form $V_{1}$ with the desired properties.

On the other hand, if an unchosen component of $T_{1}$ has a non-2-sphere boundary component $F$ in $\operatorname{Int}(L)$, then $\pi_{1}(L)$ cannot be a free group. But of more importance is the fact that the union of the other components of $T_{1}$ satisfy properties (2) and (3) of Lemma 1 with respect to the open submanifold composed of $L$ with an open collar in $S$ on $\partial L$. Thus we can apply Lemma 2 to obtain a punctured product between $F$ and a boundary component of the chosen components of $T_{1}$. This punctured product can be added to $T_{1}$ to reduce the number of non-2-sphere components of $\partial T_{1}$. This contradicts the fact that $S$ satisfies property (5) of Lemma 1 . We thus see we can assume that all the components of $T_{1}$ are chosen. To complete this stage of the construction, add the appropriate 1-handles to obtain the specified epimorphism.

Stage 5. In case 1, some 1-handles are cut and in case 2 some 1-handles are rerouted. This will yield $U_{1}$ with the desired properties. We can assume that the 1-handles of $U_{1}$ are disjoint from the 1-handles of $V_{1}^{\prime}$. Then the 1-handles of $V_{1}^{\prime}$ can be added to $U_{1}$ to form $V_{1}$ with the desired properties.

Let $\alpha_{j}$ be the core of any one of the extra 1-handles of $K_{1}$. Let $d_{j}: D^{2}$ $\rightarrow$ Int $(L)$ be a singular disk that determines a homotopy, relative to $\partial \alpha_{j}$, of $\alpha_{j}$ to a spanning arc of $U_{1}$. Assume $d_{j}$ is in general position with respect to $\partial T_{1}$. Assume also that each component of the inverse image of the 1-handles of $Y_{1}$ is a subdisk of $D^{2}$ that $d_{j}$ maps homeomorphically either to a meridional disk of a 1-handle or to a disk bounded by a core of a 1-handle and an arc in the boundary of the 1-handle. Since $\partial T_{1}$ is incompressible, we can eliminate any simple closed curves of $d_{j}^{-1}\left(\partial T_{1}\right)$. Next cut $D^{2}$ along the spanning arcs of $d_{j}^{-1}\left(\partial T_{1}\right)$, and consider the subdisk containing the arc of $\partial D^{2}$ that is mapped to $\alpha_{j}$. Redefine $d_{j}$ to be the restriction to a regular neighborhood of this subdisk. Now $d_{j}^{-1}\left(V_{1}\right)$ consists of disks in Int $\left(D^{2}\right)$ that map homeomorphically to meridional disks of 1 -handles of $V_{1}$, and of a collar on $\partial D^{2}$. This collar can be cut apart into a collection of disks such that each meets $\partial D^{2}$ in an arc 
and such that one of the disks maps into the extra 1-handle containing $\alpha_{j}$ while the others map into $T_{1}$ or 1-handles of $U_{1}$.

Construct such a map for each of the extra 1-handles of $V_{1}$, and put them in general position with respect to each other. The singular set of the union of these maps will then consist of branch points, double lines, and triple points. Adjust the maps so that no branch point is mapped into $V_{1}$. By the techniques of Smythe [15], we can assume that the image of each double line is an arc with at least one endpoint on a core of one of the extra 1-handles of $V_{1}$. By these techniques we can also eliminate triple points. This yields $D_{1}$ with the desired properties.

Let us now proceed with the construction of $T_{i+1}, U_{i+1}, V_{i+1}$ and $D_{i+1}$, assuming inductively that $T_{i}, U_{i}, V_{i}$ and $D_{i}$ have been constructed. Let $X_{i+1}$ $=V_{i} \cup D_{i} \cup B_{i+1}$ and $Y_{i+1}=S \cup T_{i}$. We know that $X_{i+1}$ can be engulfed by first adding to $S$ a punctured collar on $\partial S$ and then attaching certain 1handles to the boundary of the resulting submanifold. We can assume that $Y_{i+1}$ is contained in $S$ with the punctured collar. Let $T_{i+1}$ be the part of this punctured collar that is in $L$. Then $T_{i+1}$ is a punctured collar in $L$ on $\partial L$. Since $\partial T_{i}-\partial L$ is incompressible in $T_{i+1}$, it follows that $T_{i+1}$ is equal to $T_{i}$ with a punctured collar in $\mathrm{cl}\left(L-T_{i}\right)$ on $\partial T_{i}-\partial L$. As in the construction of $T_{1}$, we can assume that no component of $\mathrm{cl}\left(L-T_{i+1}\right)$ is compact.

By adding certain 1-handles to $T_{i+1}$ we know we can obtain a submanifold $V_{i+1}^{\prime}$ of $L$ that contains $X_{i+1}$. Add 1-handles to $T_{i+1}$ that are homotopically parallel to the 1-handles of $U_{i}$. To obtain these, notice that since $S$ is an incompressible nucleus with a maximal number of non-simply-connected complementary domains, the punctures of $T_{i+1}$ must be simply connected. Use this fact to push the 1-handles of $U_{i}$ out of the punctures of $T_{i+1}$. Assume that these 1-handles are contained in fibers of $T_{i+1}$ that miss the boundaries of these punctures. Then push these 1 -handles out of the punctured collar cl $\left(T_{i+1}-T_{i}\right)$ except near the attaching disks, where each 1-handle will intersect $T_{i+1}$ in two 3-cells that are vertical with respect to the fibers of $T_{i+1}$. Assume these 1-handles are disjoint from the 1-handles of $V_{i+1}^{\prime}$. This will give $U_{i+1}$ with the desired properties. Let $V_{i+1}$ be $U_{i+1}$ with the 1-handles of $V_{i+1}^{\prime}$ attached. The construction of the singular disks $D_{i+1}$ is similar to the construction of $D_{i}$.

We will now begin to use the $\mathbf{R}$ factor. We first want to remove singularities from $D_{i}$. Let $\alpha$ be the image of a pair of double lines. The arc $\alpha$ leads from the core of an extra 1-handle $h$ of $V_{i}$ to a branch point, to just inside $T_{i}$, or to a core of some 1-handle of $V_{i}$. In one of the singular disks there is a subdisk that is mapped homeomorphically to a meridional disk of $h$ and contains an initial segment of one of the double lines. Push a neighborhood of this subdisk slightly into the future. Follow along the double line, continue pushing, but decrease the amount of the push so that the disk is not moved at the branch 
point if one is reached, or in $V_{i}$ in the other cases. If a branch point is reached, the pair of double lines as well as the branch point will have been eliminated from the singular set. If $T_{i}$ is reached, the part of the double line mapping outside $T_{i}$ will have been eliminated from the singular set. If a 1-handle of $V_{i}$ is reached, then at the end of the double line we have been pushing along or else at the end of the double line paired with this one, there will be a subdisk mapped homeomorphically to a meridional disk of the 1-handle. In the first case push a neighborhood of the subdisk into the future. In the second case push a neighborhood of the subdisk into the past. In either case this eliminates the pair of double lines from the singular set.

After the above operations have been repeated for each pair of double lines, the singularities in each disk $d_{j}: D^{2} \rightarrow L$ will have been eliminated except inside the component of $d_{j}^{-1}\left(U_{i}\right)$ that intersects $\partial D^{2}$. The restriction of $d_{j}$ to the collar component of $d_{j}^{-1}\left(V_{i}\right)$ on $\partial D^{2}$ is unchanged by these pushes.

Notice that this process also eliminates some of the intersections of the singular disks with the 1-handles of $V_{i}$. The remaining such intersections are disjoint from the image of the original singular set. Thus these can be eliminated simply by pushing a neighborhood of the subdisk involved slightly into the future.

The pushes described above can be performed so that the image of the disks is in Int $\left(V_{i+1} \times I_{i+1}\right)$. Expand $V_{i}$ to $V_{i} \times I_{i}$ and push these disks within Int $\left(V_{i+1} \times I_{i+1}\right)$ so as to avoid creating any new intersections. The disks determine homotopies of the cores of the extra 1-handles of $V_{i}$ into $U_{i}$. Thus a regular neighborhood $\mathscr{D}_{i}$ of them in $V_{i+1} \times I_{i+1}$ can be used to define an isotopy of $L \times \mathbf{R}$ that moves points only in a neighborhood of $\mathscr{D}_{i}$ and collapses $\left(V_{i} \times I_{i}\right) \cup \mathscr{D}_{i}$ onto $U_{i} \times I_{i}$. Let $f_{i}$ denote the final stage of this isotopy.

We also want to consider the corresponding isotopy of $L \times \mathbf{R}$, which will move points only in a neighborhood of $\mathscr{D}_{i+1}$ and, at the final stage $f_{i+1}$, will map $\left(V_{i+1} \times I_{i+1}\right) \cup \mathscr{D}_{i+1}$ onto $U_{i+1} \times I_{i+1}$. Notice in particular that no point of $T_{i} \times I_{i}$ is moved by this isotopy. Thus in $L \times \mathbf{R}$ the images under $f_{i+1}$ of the cores of the 1-handles of $U_{i} \times I_{i}$, are homotopic relative to the endpoints to the cores of the 1-handles of $U_{i} \times I_{i}$, and hence to the extended cores of the 1handles of $U_{i+1} \times I_{i+1}$. But since $\pi_{1}\left(J_{i+1}\right) \rightarrow \pi_{1}(L)$ is an isomorphism,

$$
\pi_{1}\left(U_{i+1} \times I_{i+1}\right) \rightarrow \pi_{1}(L \times \mathbf{R})
$$

is also an isomorphism. Thus the homotopies also occur in $U_{i+1} \times I_{i+1}$. Since $\partial T_{i}$ is incompressible, the inclusions of components of $\partial T_{i} \times I_{i+1}$ into $U_{i+1}$ $\times I_{i+1}$ induce monomorphisms on fundamental groups. Thus we can further restrict the homotopies to occur in $\mathrm{cl}\left(U_{i+1}-T_{i}\right) \times I_{i+1}$. Since we have homotopies of arcs in a 4-manifold, we know that there is an isotopy of $L \times \mathbf{R}$ such that only points in $\mathrm{cl}\left(U_{i+1}-T_{i}\right) \times I_{i+1}$ are moved and, at the final stage 
$g_{i+1}$, the images under $f_{i+1}$ of the 1-handles of $U_{i} \times I_{i}$ are mapped to regular neighborhoods in $\mathrm{cl}\left(U_{i+1}-T_{i}\right) \times I_{i+1}$ of the extended cores of 1-handles of $U_{i+1} \times I_{i+1}$. Let $g_{1}$ be the identity map on $L \times \mathbf{R}$.

After we have found these isotopies and the resulting homeomorphisms $f_{i}$ and $g_{i}$ for all $i \geqslant 1$, we have the commutative diagram of Figure 2. All horizontal arrows indicate inclusions.

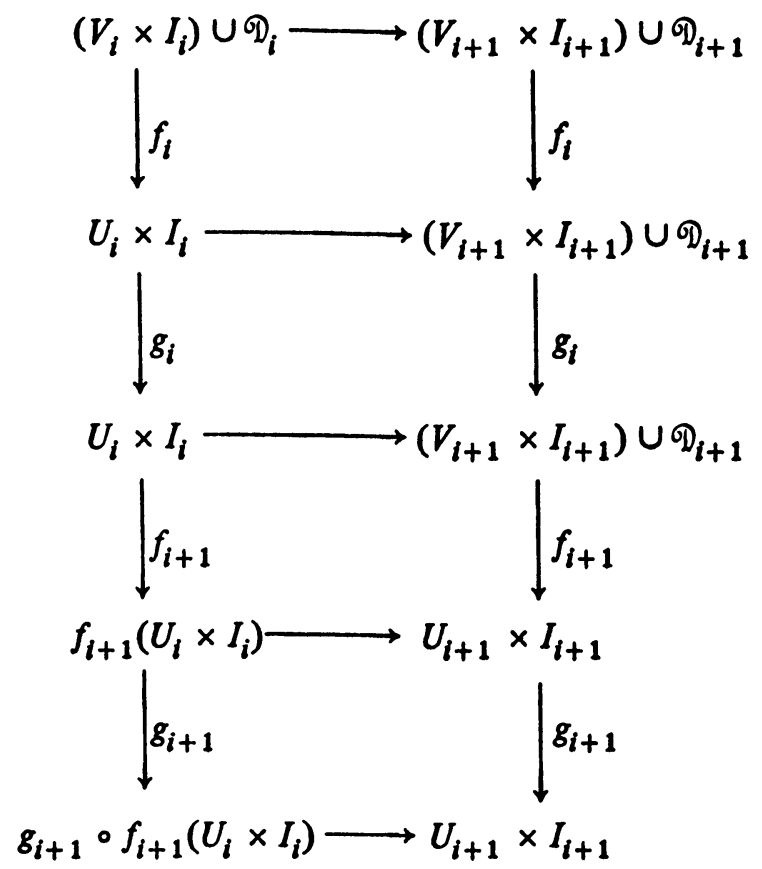

FIGURE 2

Using the fact that for each $i \geqslant 1, \mathrm{cl}\left(T_{i+1}-T_{i}\right)$ is a punctured collar on $\partial T_{i}-\partial L$, we can construct a homeomorphism

$$
h_{i}: T_{i} \times \mathbf{R} \rightarrow\left((\partial L \times[0, i])-\operatorname{Int}\left(B_{i}^{3}\right)\right) \times \mathbf{R},
$$

where $B_{i}^{3}$ is an appropriate union of disjoint 3-cells. We can assume we have the commutative diagram of Figure 3.

Notice that

$$
B_{i+1}^{3} \cap(\partial L \times[0, i]) \subseteq \operatorname{Int}\left(B_{i}^{3}\right)
$$

and that we can assume

$$
\bigcap_{j=i}^{\infty}\left(B_{j}^{3} \cap(\partial L \times[0, i])\right)
$$

is a closed, 0-dimensional set of points in $\partial L \times(0, i)$. 


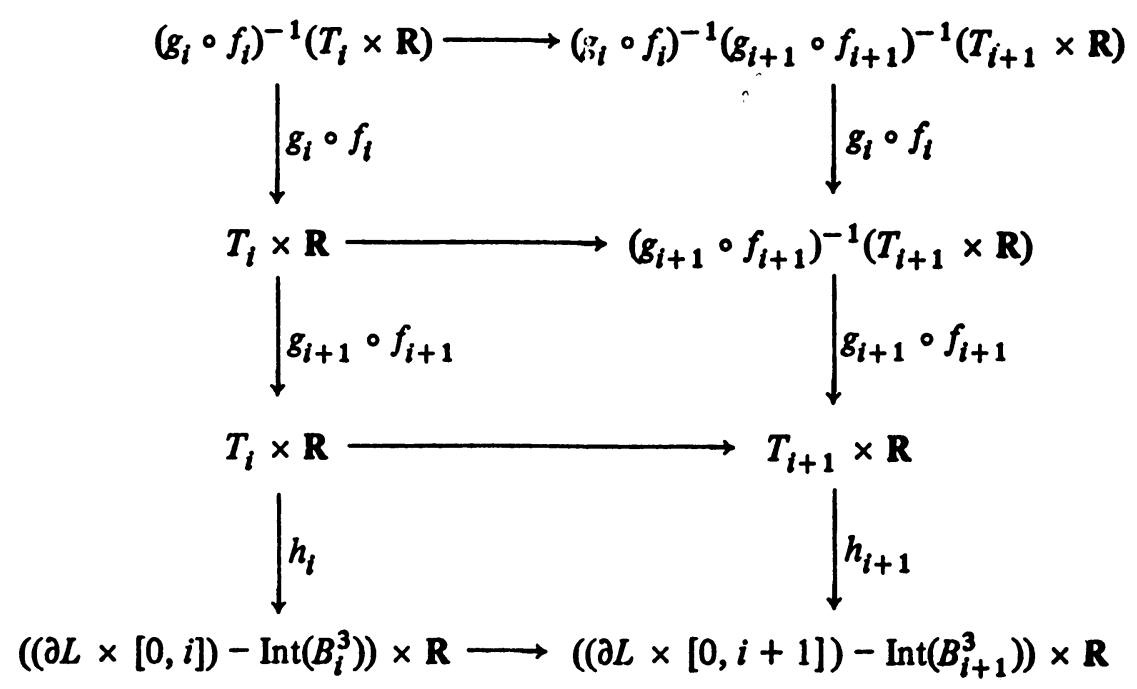

FIGURE 3

We now have for each $i \geqslant 1$ a homeomorphism

$$
\begin{aligned}
h_{i} & \circ\left(g_{i} \circ f_{i} \circ \cdots \circ g_{1} \circ f_{1}\right)\left(g_{i} \circ f_{i} \circ \cdots \circ g_{1} \circ f_{1}\right)^{-1}\left(T_{i} \times \mathbf{R}\right): \\
& \left(g_{i} \circ f_{i} \circ \cdots \circ g_{1} \circ f_{1}\right)^{-1}\left(T_{i} \times \mathbf{R}\right) \rightarrow\left((\partial L \times[0, i])-\operatorname{Int}\left(B_{i}^{3}\right)\right) \times \mathbf{R} .
\end{aligned}
$$

The union of these gives a homeomorphism of

$$
\bigcup_{i=1}^{\infty}\left(g_{i} \circ f_{i} \circ \cdots \circ g_{1} \circ f_{1}\right)^{-1}\left(T_{i} \times \mathbf{R}\right)
$$

onto $((\partial L \times[0, \infty))-P) \times \mathbf{R}$ where $P$ is a tame, closed, 0 -dimensional set of points in $\partial L \times(0, \infty)$.

Finally we must see that the remainder of $L \times \mathbf{R}$ forms open 1-handles that can be mapped to open 1-handles attached to

$$
((\partial L \times[0, \infty))-P) \times \mathbf{R} \text {. }
$$

Now $U_{1} \times I_{1}$ is the union of $T_{1} \times I_{1}$ and 1-handles with attaching disks $D_{1}^{2} \times I_{1}$, where $D_{1}^{2}$ consists of disjoint pairs of disks in the components of $\partial T_{1}-\partial L$ that are not boundaries of the punctures. Extend $h_{1}$ to map these 1handles to 1 -handles attached to $(\partial L \times\{1\}) \times \mathbf{R}$. Assume the intersection of these 1-handles with $((\partial L \times[0, \infty))-P) \times \mathbf{R}$ is $\left(D_{1}^{2} \times[1, \infty)\right) \times I_{1}$ where $D_{1}^{2}$ also denotes disks corresponding to the attaching disks in $(\partial L \times\{1\}) \times \mathbf{R}$. With this extension of $h_{1}$, we have defined a homeomorphism $h_{1} \circ g_{1}$ - $f_{1} \mid\left(V_{1} \times I_{1}\right) \cup \mathscr{D}_{1}$ on $\left(V_{1} \times I_{1}\right) \cup \mathscr{D}_{1}$.

Assume inductively that we have extended $h_{i}$ to $U_{i} \times I_{i}$ so that the images of the 1-handles follow the fibers of the collar $(\partial L \times[i, \infty)) \times \mathbf{R}$ and so that the 
homeomorphism $h_{i} \circ g_{i} \circ f_{i} \mid\left(V_{i} \times I_{i}\right) \cup \mathscr{D}_{i}$ agrees with $h_{i-1}$ on $U_{i-1} \times I_{i-1}$.

Now $U_{i+1} \times I_{i+1}$ is the union of $T_{i+1} \times I_{i+1}$ and 1-handles with attaching disks $D_{i+1}^{2} \times I_{i+1}$, where $D_{i+1}^{2}$ consists of disjoint pairs of disks in the components of $\partial T_{i+1}-\partial L$ that are not boundaries of the punctures. Recall that $g_{i+1}$ straightens the image under $f_{i+1}$ of the 1-handles of $U_{i} \times I_{i}$ so that they are regular neighborhoods of the extended cores of the corresponding 1-handles of $U_{i+1} \times I_{i+1}$. Adjust the map $g_{i+1}$ so that any point of the 1-handles of $U_{i} \times I_{i}$ that is mapped to $\left(D_{i}^{2} \times[i, i+1]\right) \times I_{i}$ by $h_{i}$ is mapped to the same point by

$$
h_{i+1} \circ g_{i+1} \circ f_{i+1} \mid\left(g_{i+1} \circ f_{i+1}\right)^{-1}\left(T_{i+1} \times \mathbf{R}\right) .
$$

Now we can extend $h_{i+1}$ to map the 1-handles of $U_{i+1} \times I_{i+1}$ to 1-handles attached to $(\partial L \times\{i+1\}) \times \mathbf{R}$. Assume the intersection of these 1-handles with $((\partial L \times[0, \infty))-P) \times R$ is

$$
\left(D_{i+1}^{2} \times[i+1, \infty)\right) \times I_{i+1},
$$

where $D_{i+1}^{2}$ also denotes disks corresponding to the attaching disks in $(\partial L \times\{i+1\}) \times \mathbf{R}$. Assume also that this extension of $h_{i+1}$ agrees with $h_{i} \circ\left(g_{i+1} \circ f_{i+1}\right)^{-1}$ on $g_{i+1} \circ f_{i+1}\left(U_{i} \times I_{i}\right)$. Then

$$
h_{i+1} \circ g_{i+1} \circ f_{i+1} \mid\left(V_{i+1} \times I_{i+1}\right) \cup \mathscr{D}_{i+1}
$$

will agree with $h_{i}$ on $U_{i} \times I_{i}$. This gives the commutative diagram of Figure 4, where $H_{i}$ and $H_{i+1}$ denote the 1-handles that have been added.

Since we have $L \times \mathbf{R}=\cup_{i=1}^{\infty}\left(\left(V_{i} \times I_{i}\right) \cup \mathscr{D}_{i}\right)$, the union of the homeomorphisms $h_{i} \circ\left(g_{i} \circ f_{i} \circ \cdots \circ g_{1} \circ f_{1}\right) \mid\left(V_{i} \times I_{i}\right) \cup \mathscr{D}_{i}$ gives a homeomorphism onto the product of $\mathbf{R}$ and $(\partial L \times[0, \infty))-P$ with certain open 1-handles attached.

As remarked at the beginning of the proof, this construction can be performed for any component of $M-\operatorname{Int}(S)$. Hence we can put all these homeomorphisms together with a map from $S \times \mathbf{R}$ to another copy of $S \times \mathbf{R}$ to obtain a homeomorphism from $M \times \mathbf{R}$ onto the product of $\mathbf{R}$ and $S \cup((\partial S \times[0, \infty))-P)$ with certain open 1 -handles attached. Here $P$ is a tame, closed, 0 -dimensional subset of $\partial S \times(0, \infty)$ obtained from the corresponding subsets of the open collars on the boundary components of the complementary domains of $S$. Let $U$ denote $S$ with the appropriate 1-handles attached to $\partial S$ and let $C$ denote an open collar on $\partial U$. Then $M \times \mathbf{R}$ is homeomorphic to $(U \cup(C-P)) \times \mathbf{R}$.

The proofs of the following two corollaries are based on some of the constructions from the proof of Theorem 4.

COROLlaRY. In the situation stated in Theorem 4, suppose also that $\pi_{1}(M)$ is indecomposable and non-infinite-cyclic. Then there is an isotopy that moves no 

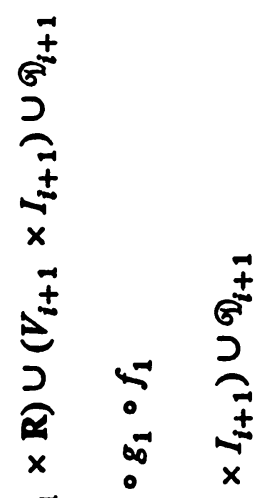

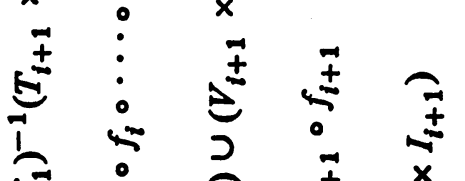
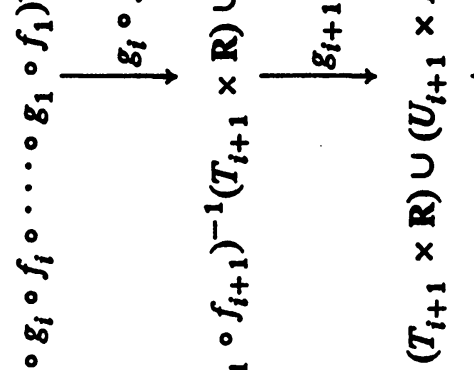

$\pm$

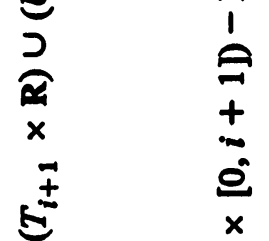

$\underbrace{ \pm}_{0}$

e्छ

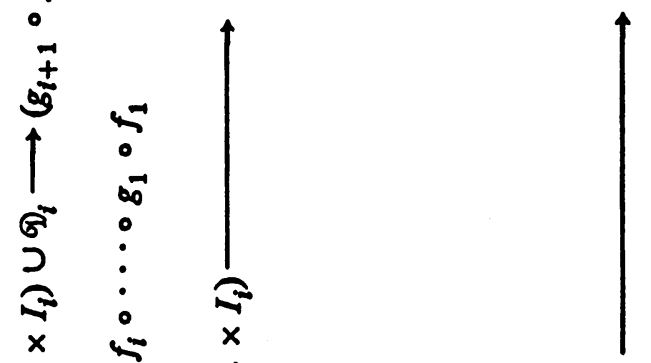

\begin{tabular}{ll}
0 & 0 \\
2 & $\infty$ \\
\hline
\end{tabular}

$\frac{2}{2}$

$\mathbb{E}^{\infty} \quad \mathbb{E}^{-}$

है

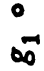

0

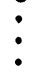

0

4 
points of $S \times \mathbf{R}$, between the identity embedding of $M \times \mathbf{R}$ into $M \times \mathbf{R}$ and an embedding of $M \times \mathbf{R}$ onto $(S \cup C) \times \mathbf{R}$ where $C$ is an open collar on $\partial S$ in $M$ - Int $(S)$ with a tame, closed, 0-dimensional set of points deleted from its interior.

Proof. Since $\pi_{l}(M)$ is indecomposable, $S$ is connected. Indeed by property (3) of Lemma 1, all of $\pi_{1}(M)$ is conjugate to a subgroup of the fundamental group of a single component of $S$. In particular no other component of $S$ can have a non-2-sphere boundary component. Otherwise Lemma 2 would give a punctured product between two non-2-sphere components of $\partial S$. This could be added to $S$, contrary to property (5). But now notice that property (6) eliminates the possibility that there are other components of $S$ with only 2 sphere boundary components.

From the fact that $\pi_{1}(M)$ is conjugate to a subgroup of $\pi_{1}(S)$, it follows that the map $\pi_{1}(S) \rightarrow \pi_{1}(M)$ induced by inclusion is an isomorphism. Hence in the proof of Theorem 4, no 1-handles are needed to generate $\pi_{1}(L)$. Therefore $U_{i}=T_{i}$ for all $i \geqslant 1$. Let us define an isotopy of $L \times \mathbf{R}$ in the interval $[(i-1) / i, i /(i+1)]$ by using the isotopy that deforms $\left(V_{i} \times I_{i}\right) \cup \mathscr{D}_{i}$ to $U_{i} \times I_{i}$ $=T_{i} \times I_{i}$. Since the isotopy that deforms $\left(V_{i+1} \times I_{i+1}\right) \cup \mathscr{D}_{i+1}$ does not move points of $T_{i} \times I_{i}$, we see that each point of $L \times \mathbf{R}$ is moved only by a finite number of these isotopies. Therefore we can put these isotopies together with the limiting homeomorphism to define an isotopy of $L \times \mathbf{R}$.

As in Theorem 4, the desired isotopy of $M \times \mathbf{R}$ can be defined by using the identity isotopy on $S \times \mathbf{R}$ and the above isotopies in the components of $(M-\operatorname{Int}(S)) \times \mathbf{R}$.

Corollary. Suppose $P$ and $P^{*}$ are Cantor sets embedded in the 3-sphere $S^{3}$ so that their complements have finitely generated fundamental groups. Then the complements are simply connected and $\left(S^{3}-P\right) \times \mathbf{R}$ is homeomorphic to $\left(S^{3}-P^{*}\right) \times \mathbf{R}$.

Proof. It suffices to prove this corollary in the case that $P^{*}$ is a tame Cantor set.

To show that $\pi_{1}\left(S^{3}-P\right) \cong(0)$, consider the following composition. Include $S^{3}-P$ as the 0 -level of $\left(S^{3}-P\right) \times \mathbf{R}$. Follow this by the homeomorphism given in Theorem 4 of $\left(S^{3}-P\right) \times \mathbf{R}$ onto $\left(U \cup\left(C-P^{\prime}\right)\right) \times \mathbf{R}$ where $P^{\prime}$ denotes the tame, 0-dimensional subset of the open collar $C$ on $\partial U$. Then apply the projection onto $U \cup\left(C-P^{\prime}\right)$ followed by the inclusion into $U \cup C$. Finally apply a retraction of $U \cup C$ onto $U$.

$$
\begin{aligned}
S^{3}-P & \rightarrow\left(S^{3}-P\right) \times \mathbf{R} \rightarrow\left(U \cup\left(C-P^{\prime}\right)\right) \times \mathbf{R} \\
& \rightarrow U \cup\left(C-P^{\prime}\right) \rightarrow U \cup C \rightarrow U .
\end{aligned}
$$

Notice that each map of this composition induces an isomorphism between 
the first homology groups of the spaces involved. Thus $H_{1}\left(S^{3}-P\right) \cong H_{1}(U)$. Together with the fact that $H_{1}\left(S^{3}-P\right) \cong H_{1}\left(S^{3}\right) \cong(0)$, this gives $H_{1}(U)$ $\cong(0)$. Since $U$ is homeomorphic to a compact submanifold of $S^{3}, U$ must be simply connected. The maps of the above composition also induce isomorphisms between fundamental groups. Therefore $\pi_{1}\left(S^{3}-P\right) \cong \pi_{1}(U) \cong(0)$.

Now since $S^{3}-P$ is simply connected, we can choose a 3-cell $S$ of $S^{3}-P$ as an incompressible nucleus with a maximal number of non-simply-connected complementary domains. Then $T_{i}$ is homeomorphic to $(\partial S \times[0, i])$ - Int $\left(B_{i}^{3}\right)$ where $B_{j}^{3}$ is a union of disjoint 3-cells in $\partial S \times(0, i)$. Since $\partial S \times\{i\}$ is a 2 -sphere in $S^{3}$, let us add to $B_{i}^{3}$ the 3-cell complementary domain of $\partial S \times\{i\}$ not containing $S$. Then $S \cup T_{i}$ is homeomorphic to $S^{3}-\operatorname{Int}\left(B_{i}^{3}\right)$. We also have $B_{i+1}^{3} \subseteq \operatorname{Int}\left(B_{i}^{3}\right)$, and we can assume the $B_{i}^{3}$ are chosen so that $\bigcap_{i=1}^{\infty} B_{i}^{3}$ is a tame Cantor set $P^{*}$. Thus the union of the homeomorphisms

$$
\begin{aligned}
& h_{i} \circ\left(g_{i} \circ f_{i} \circ \cdots \circ g_{1} \circ f_{1}\right) \mid\left(g_{i} \circ f_{i} \circ \cdots \circ g_{1} \circ f_{1}\right)^{-1}\left(T_{i} \times \mathbf{R}\right): \\
& \left(g_{i} \circ f_{i} \circ \cdots \circ g_{1} \circ f_{1}\right)^{-1}\left(T_{i} \times \mathbf{R}\right) \rightarrow\left(S^{3}-\operatorname{Int}\left(B_{i}^{3}\right)\right) \times \mathbf{R}
\end{aligned}
$$

as defined in the proof of Theorem 4 gives a homeomorphism from $\left(S^{3}-P\right)$ $\times \mathbf{R}$ onto $\left(S^{3}-P^{*}\right) \times \mathbf{R}$.

3. The missing boundary problem. In a recent paper [19], T. W. Tucker has proved the following theorem concerning almost compact 3-manifolds, that is, 3-manifolds $M$ that are homeomorphic to $N-L$ where $N$ is a compact 3manifold and $L$ is a closed subset of $\partial N$.

THEOREM. Suppose $M$ is a connected, $P^{2}$-irreducible 3-manifold. Then $M$ is almost compact if and only if for any compact polyhedron $C$ in $M$, the fundamental group of each component of $M-C$ is finitely generated.

Theorem 5 shows that in order to test whether $M$ is almost compact, it suffices to consider only polyhedra $C$ that are simple closed curves.

Consider the closed complementary domain of a 3-cell constructed on the basis of a Fox-Artin arc (see Example 1.2 of [2]). After removing the wild point, we have a 3-manifold that is not almost compact, although its interior is an open 3-cell. This example shows that we cannot restrict our consideration in Theorem 5 to simple closed curves in the interior of the manifold.

THEOREM 5. SUppose $M$ is a connected, irreducible 3-manifold such that for any polyhedral simple closed curve $J$ in $M, \pi_{1}(M-J)$ is finitely generated. Then for any compact polyhedron $C$ in $M$, the fundamental group of each component of $M-C$ is finitely generated.

Proof of Theorem 5. Assume first that $C$ is a compact, connected submanifold of $M$. Then $C$ can be obtained by adding 1-handles to disjoint 0 - 
handles, and then attaching 2-handles to the boundary of the resulting submanifold. We want to build $C$ by adding these handles and to check at various stages that the complementary components have finitely generated fundamental groups.

Let $C^{\prime}$ be the union of the 0 -handles and the 1-handles. Then $C^{\prime}$ is a regular neighborhood of a connected 1-complex. By induction on the number of edges of this 1-complex, it is easy to check that there is an even number of vertices that are contained in an odd number of edges. Thus we can attach 1-handles to $C^{\prime}$ to form a submanifold $C^{\prime \prime}$ that is a regular neighborhood of a 1-complex with all vertices contained in an even number of edges. We can choose such a 1-complex $G$ with the additional properties that all vertices are contained in either two or four edges, and for any vertex in $\partial M$ that is contained in four edges, the four edges are also in $\partial M$.

Consider disjoint 3-cell regular neighborhoods $B_{j}$ of the vertices of $G$ that are contained in four edges. For such a vertex in $\partial M, B_{j} \cap \partial M$ will consist of a disk in $\partial B_{j}$, which will contain the intersection of $B_{j}$ with the four edges. Modify $G$ in these 3-cells as indicated in Figure 5.

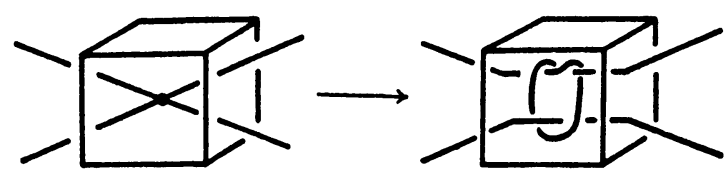

for a vertex in $\operatorname{Int}(M)$

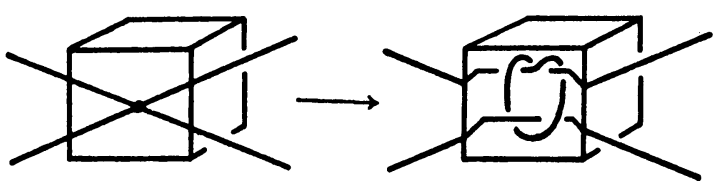

for a vertex in $\partial M$

FIGURE 5

This will yield a union of disjoint simple closed curves in $M$. By altering the choice as to which pairs of edges are joined together in the appropriate $B_{j}$, we can reduce the number of components until we have only one simple closed curve $J$. This is related to the remark by Euler concerning the problem of the seven bridges of Königsberg: a connected graph contains a closed path passing through each edge exactly once if and only if every vertex is contained in an even number of edges. 
Notice that for each $j$, the map $\pi_{1}\left(\partial B_{j}-J\right) \rightarrow \pi_{1}\left(B_{j}-J\right)$ induced by inclusion is a monomorphism. Otherwise the loop theorem would give a nontrivial simple closed curve in $\partial B_{j}-J$ that bounds a disk properly embedded in $B_{j}-J$. Such a simple closed curve could not separate the endpoints of either arc of $B_{j} \cap J$ in $\partial B_{j}$. Therefore the disk would separate $B_{j}$ into two 3-cells, one containing the knotted arc of $B_{j} \cap J$ and the other containing the unknotted arc of $B_{j} \cap J$. In particular, this would show that in the complement of the knotted arc there is a homotopy of the unknotted arc into $\partial B_{j}$. This contradicts Bing's Lemma 6 of [1].

Let $B$ denote the union of the 3-cells $B_{j}$. Suppose that cl $(\partial B-\partial M)-J$ is compressible in $M-J$. Let $D$ be a compressing disk. Let $B_{j}$ be the 3-cell of $B$ that contains $\partial D$. By our choice of $G$ it follows that $B_{j} \subseteq$ Int $(M)$. By the result of the previous paragraph, $D$ must be properly embedded in $\mathrm{cl}(M-B)$ $-J$. Now $\partial D$ separates $\partial B_{j}$ into two disks $E_{1}$ and $E_{2}$. Since $M$ is irreducible, $D \cup E_{1}$ and $D \cup E_{2}$ bound 3-cells. If the 3-cell bounded by $D \cup E_{1}$ does not meet $B_{j}$ only along $E_{1}$, then it contains $B_{j}$. In this case, $D \cup E_{2}$ will be a 2sphere in this 3-cell, and hence it will bound a 3-cell meeting $B_{j}$ only along $E_{2}$. In either case we have found a 3 -cell $C^{3}$ meeting $B_{j}$ only along $E$, which is $E_{1}$ or $E_{2}$. Since $\partial D$ is nontrivial in $\partial B_{j}-J, E$ contains some but not all of the four points of $\partial B_{j} \cap J$. Therefore $C^{3}$ must contain one of the arcs of $J$ - Int $\left(B_{j}\right)$; incidentally, $E$ contains exactly two of the points of $\partial B_{j} \cap J$. Replace $B_{j}$ by the 3-cell $C^{3} \cup B_{j}$ and remove from the collection of 3-cells under consideration any that are contained in the interior of the new $B_{j}$. Now $\partial B_{j}$ separates $J$ into two subarcs, so cl $\left(\partial B_{j}-\partial M\right)-J$ is incompressible in $M-J$.

We can repeat this process until, after a finite number of applications, cl $(\partial B-\partial M)-J$ is incompressible in $M-J$. By hypothesis, $\pi_{1}(M-J)$ is finitely generated. By Lemma 4 , the complementary domain $\mathrm{cl}(M-B)$ $-J$ of $\mathrm{cl}(\partial B-\partial M)-J$ has a finitely generated fundamental group. The fundamental groups of the components of $B-G$ are also finitely generated. Therefore, by Van Kampen's theorem, $\pi_{1}(M-G)$ is finitely generated. It follows that $\pi_{1}\left(M-C^{\prime \prime}\right)$ is finitely generated. Removing the 1-handles of $C^{\prime \prime}$ that were added to $C^{\prime}$ is essentially equivalent to adding 2-handles to cl $\left(M-C^{\prime \prime}\right)$. Therefore $\pi_{1}\left(M-C^{\prime}\right)$ is also finitely generated. To obtain $C$, we need to attach 2-handles to $C^{\prime}$. This is essentially equivalent to cutting 1handles from $\mathrm{cl}\left(M-C^{\prime}\right)$. Since free-product factors of a finitely generated group are finitely generated, it follows that $\pi_{1}(M-C)$ is finitely generated.

If $C$ is not connected, we can join the components of $C$ with appropriate 1handles attached to $\partial C$. The complementary components of the resulting submanifold will have finitely generated fundamental groups. As we saw previously, removing these 1-handles will preserve this property.

Finally if $C$ is not a submanifold of $M$, simply notice that the complement 
of $C$ is homotopy equivalent to the complement of a regular neighborhood of C.

\section{REFERENCES}

1. R. H. Bing, Necessary and sufficient conditions that a 3-manifold be $S^{3}$, Ann. of Math. (2) 68 (1958), 17-37. MR 20 \#1973.

2. Ralph H. Fox and Emil Artin, Some wild cells and spheres in three-dimensional space, Ann. of Math. (2) 49 (1948), 979-990. MR 10, 317.

3. D. E. Galewski, J. G. Hollingsworth and D. R. McMillan, Jr., On the fundamental group and homotopy type of open 3-manifolds, General Topology and Appl. 2 (1972), 299-313. MR 47 \#5880.

4. Wolfgang Haken, Some results on surfaces in 3-manifolds, Studies in Modern Topology, Math. Assoc. Amer., Prentice-Hall, Englewood Cliffs, N. J. 1968, pp. 39-98. MR 36 \# 7118.

5. William S. Massey, Algebraic topology: An introduction, Harcourt, Brace, and World, New York, 1967. MR 35 \#2271.

6. D. R. McMillan, Jr., Cartesian products of contractible open manifolds, Bull. Amer. Math. Soc. 67 (1961), 510-514. MR 24 \#A1132.

7. Canonical neighborhoods in three-manifolds (Topology Seminar, Wisconsin, 1965), Princeton Univ. Press, Princeton, N. J., 1966, pp. 61-67. MR 34 \# 1974; erratum, 35, p. 1577.

8. - A finiteness theorem for open three-manifolds, Proc. Topology Conf. (Univ. of Oklahoma, 1972), University of Oklahoma, Norman, 1972, pp. 147-167.

9. D. R. McMillan, Jr. and E. C. Zeeman, On contractible open manifolds, Proc. Cambridge Philos. Soc. 58 (1962), 221-224. MR 25 \#2591.

10. Robert Messer, Three-dimensional manifolds and compactness, Ph.D. thesis, Univ. of Wisconsin, Madison, 1975.

11. - Open three-dimensional manifolds with finitely generated fundamental groups, Bull. Amer. Math. Soc. 82 (1976), 89-91.

12. C. P. Rouke and B. J. Sanderson, Introduction to piecewise-linear topology, Springer-Verlag, New York, 1972. MR 50 \#3236.

13. G. P. Scott, Finitely generated 3-manifold groups are finitely presented, J. London Math. Soc. (2) 6 (1973), 437-440.

14. Compact submanifolds of 3-manifolds, J. London Math. Soc. (2) 7 (1973), 246-250. MR 48 \#5080.

15. N. Smythe, Handlebodies in 3-manifolds, Proc. Amer. Math. Soc. 26 (1970), 534-538. MR 41 \#9236.

16. John Stallings, On the loop theorem, Ann. of Math. (2) 72 (1960), 12-19. MR 22 \# 12526.

17. - On fibering certain 3-manifolds, Topology of 3-Manifolds and Related Topics

(Proc. Univ. of Georgia Inst., 1961), Prentice-Hall, Englewood Cliffs, N. J., 1962, pp. 95-100. MR 28 \# 1600.

18. T. W. Tucker, Boundary-reducible 3-manifolds and Waldhausen's theorem, Michigan Math. J. 20 (1973), 321-327. MR 48 \# 12537.

19. - Non-compact 3-manifolds and the missing-boundary problem, Topology 13 (1974), 267-273. MR 50 \#5801.

20. Friedhelm Waldhausen, Eine Verallgemeinerung des Schleifensatzes, Topology 6 (1967), 501-504. MR 36 \#3366.

21. - On irreducible 3-manifolds which are sufficiently large, Ann. of Math. (2) 87 (1968), 56-88. MR 36 \# 7146.

22. J. H. C. Whitehead, A certain open manifold whose group is unity, Quart. J. Math. Oxford Ser. 6 (1935), 268-279.

Department of Mathematics, University of Wisconsin, Madison, Wisconsin 53706

Current address: Department of Mathematics, Dartmouth College, Hanover, New Hampshire 03755 